\title{
DISCURSOS JUVENILES DESDE UN CASO EN LA CIUDAD DE BAYAMO-CUBA. REFLEXIONES EN TIEMPOS DE LA COVID-19
}

\section{YOUTH SPEECHES FROM A CASE IN THE CITY OF BAYAMO IN CUBA. REFLECTIONS IN TIMES OF COVID-19}

\author{
Reynier Espinosa García ${ }^{1}$ \\ Elizabeth Guerra Roblejo² \\ Maylin Yero Perea ${ }^{3}$ \\ Alisa Natividad Delgado Tornés ${ }^{4}$
}

Recibido: 2020-02-05 / Revisado: 2020-03-15 / Aceptado: 2020-05-05 / Publicado: 2020-07-01

Forma sugerida de citar: Espinosa-García, R., Guerra-Roblejo, E., Yero-Perea, M. y Delgado-Tornés, A. N. (2020). Discursos juveniles desde un caso en la ciudad de Bayamo-Cuba. Reflexiones en tiempos de la COVID19. Retos de la Ciencia. 4(9), pp. 57-65. doi.org/10.53877/rc.4.9.20200701.06

\section{RESUMEN}

Los discursos son mecanismos viables para el estudio de las diferentes posiciones con relación a determinados objetos y fenómenos. Como forma de práctica social, los discursos remiten a agentes, prácticas e instituciones que se estructuran en contextos determinados. Este estudio preliminar, de carácter exploratorio, analiza las diversas posiciones discursivas de jóvenes, entre 19 y 30 años, frente a la situación social emergente en el contexto de la COVID-19. Se llevó a cabo desde el enfoque del análisis sociológico de discurso, en la perspectiva sociohermenéutica. El objeto de estudio fueron los discursos provocados en 13 jóvenes bayameses residentes en el área entre el Centro Histórico Urbano, calle 3 de Camilo Cienfuegos, y la periferia Norte, calle 24 de Ciro Redondo, mediante la técnica de la entrevista semiestructurada. Los resultados muestran algunos posicionamientos discursivos de matices positivo, negativo y neutro en relación con

\footnotetext{
${ }^{1}$ Licenciada en Historia del Arte. Máster en Historia y Cultura en Cuba. Aspirante a Investigador de la Casa de la Nacionalidad. Doctorando en Ciencias Sociológicas de la Universidad de Oriente. Santiago de Cuba. Cuba. Email: reynierespinosa84@gmail.com / https://orcid.org/0000-0001-8842-880x

${ }^{2}$ Licenciada en Psicología. Máster en Desarrollo Socioeconómico Local. Aspirante a Investigador de la Casa de la Nacionalidad. Santiago de Cuba-Cuba. E-mail: eguerraroblejo@gmail.com / https://orcid.org/0000-00026449-8990

${ }^{3}$ Licenciada en Historia del Arte. Doctora en Ciencias Sociológicas. Instituto María Reina. Profesora del Doctorado en Ciencias Sociológicas de la Universidad de Oriente. Santiago de Cuba-Cuba. E-mail: myeroperea@gmail.com / https://orcid.org/0000-0003-1610-2194

${ }^{4}$ Licenciada en Ciencias Políticas. Doctora en Ciencias Filosóficas. Profesora Titular en la Universidad de Granma. Grama-Cuba. Profesora y Miembro del Comité del Doctorado en Ciencias Sociológicas de la Universidad de Oriente. Santiago de Cuba-Cuba. E-mail: alisadelgado@nauta.cu / https://orcid.org/0000-0001$9061-2585$
} 
la percepción de riesgo a contraer la pandemia, los principales cambios asociados con las normas higiénicas, las iniciativas locales, la protección de los grupos vulnerables y la valoración de gestión de la información que reciben. Se evidencian las posiciones de los jóvenes participantes frente a un conjunto de retos que se derivan de su percepción y actitud ante esta pandemia, que inciden en su integración plena ante esta nueva realidad.

Palabras claves: análisis del discurso, posiciones discursivas; COVID-19, discurso juvenil.

\section{ABSTRACT}

Discourses are viable mechanisms for the study of different positions in relation to certain objects and phenomena. As a form of social practice, discourses refer to agents, institutions and practices that are structured in certain contexts. This preliminary exploratory study analyzes the diverse discursive positions of young people in the face of the emerging social situation in the context of COVID-19. It was carried out from the perspective of the sociological analysis of discourse, in the perspective of socio-hermeneutical analysis. The object of study consisted of the provoked speeches of 13 young bayameses residing in an interval between the Urban Historic Center (calle 3 de Camilo Cienfuegos) and the northern periphery (calle 24 de Ciro Redondo), using the semi-structured interview technique. The results show some discursive positions regarding the perception of risk of contracting the pandemic, the main changes associated with hygiene standards, local initiatives, the protection of vulnerable groups, and the management evaluation of the information they receive.

Key words: discourse analysis, discursive positions, COVID- 19, youth discourse.

\section{INTRODUCCIÓN}

El análisis sociológico del discurso permite comprender las prácticas sociales que se producen y reproducen en diferentes grupos, en un determinado contexto social. En medio del despliegue de la pandemia de la COVID-19 es importante develar las prácticas juveniles y su discurso alrededor de los acontecimientos, así como los valores que prevalecen en la sociedad bayamesa.

Desde el 11 de marzo con la emergencia de casos de la COVID-19 la sociedad cubana muestra un panorama, en cuanto a las reacciones y percepciones de los cubanos en torno a esta enfermedad, de profundas complejidades que conducen a las ciencias sociales a reflexionar los actuales procesos que acompañan sus impactos sociales en diferentes grupos poblacionales, entre ellos, el de los jóvenes. En la actualidad, la reemergencia de los grupos juveniles en la vida social desde diferentes perspectivas coloca la juventud como uno de los grupos más abordados por las ciencias sociales en las últimas décadas en Cuba.

Por ello, se producen investigaciones interdisciplinares para estudiar este sector poblacional, sobre todo para entender y ubicar las prácticas, usos, estilos y/o hábitos en las que parte de la juventud encuentran sentidos y significados sociales y culturales (Morales, 2017; Domínguez, 2010 y 2011; Rego, 2014). De ahí que se estudien los procesos o fenómenos asociados a los jóvenes desde ellos mismos y no a partir de preconcepciones que obstaculizan y refuerzan barreras (Morales, 
2017). Tal es el caso, de los discursos sociales juveniles desde determinadas posiciones sociales que condicionan tanto su forma como su contenido. Este trabajo intenta aproximarse a la realidad social de un sector juvenil en la ciudad de Bayamo, Provincia de Granma, a través de sus discursos en el contexto de la COVID-19.

El surgimiento de la pandemia del COVID-19, calificada por Ramonet (2020) como un "hecho social total", no solo ha trastocado el conjunto de relaciones sociales, ha sacudido actores, instituciones y valores; sino que además ha provocado un estallido de los discursos sociales al alterar todos los campos semánticos de la vida social, sobre todo, lo relacionado con los comportamientos. De manera que indagar acerca de los discursos sociales en este contexto permitiría aproximarse a la situación social emergente y a los diversos posicionamientos, roles y prácticas de actores e instituciones sociales en este contexto particular.

En Cuba, aunque no se decretó el estado de emergencia social, se diseñó e implementó un Plan de Medidas para el enfrentamiento a la COVID-19 que implicó a todos los organismos de la administración central del Estado, las empresas, el sector no estatal y la población general. La ejecución de este plan a finales del mes de marzo ocasionó alteraciones en la vida cotidiana de los cubanos, sobre todo, en lo referido a las prácticas de socialización habituales por el cierre y restricciones de espacios públicos y privados, la exigencia del autocuidado y del aislamiento social. Desde el comienzo del periodo las instituciones políticas y sociales insisten en el cumplimiento de las medidas por parte de todos los sectores, haciendo hincapié en el sector juvenil y de la tercera edad.

La insistencia en el cumplimiento de las medidas en el sector juvenil se relaciona con la baja vulnerabilidad de este. Este sector poblacional, por su dinamismo, constituye un grupo social y cultural importante dentro del enfrentamiento a la pandemia, lo que implica un alto compromiso en el mantenimiento de la disciplina social. Sin embargo, reportes televisivos, fotografías en las redes sociales y la observación participante en la vida cotidiana, demuestran dos puntos de vista: por un lado, jóvenes en fiestas, en las esquinas, sin protección, poca participación en las labores domésticas y comentarios de rechazo a las medidas de aislamiento social, etc.; y por otro lado, jóvenes en brigadas de solidaridad prestando ayuda a sectores vulnerables, realizando pesquisas puerta a puerta para identificar sospechosos o enfermos, o trabajando como voluntarios en centros de aislamiento. Esta división del sector juvenil nos resultó de interés para la ejecución de un estudio interpretativo de las diversas posiciones discursivas de los jóvenes frente a la situación social emergente en el contexto de la COVID-19.

Este estudio se llevó a cabo desde el enfoque del análisis sociológico de discurso, en la perspectiva del análisis sociohermenéutico (Alonso, 2013; Ruiz y Alonso, 2019). En el presente estudio se considera el análisis sociológico de discurso (ASD) como un enfoque de investigación sociológica dentro de la Sociología actual que estudia la sociedad y las relaciones sociales mediante (el análisis de) los discursos. Se diferencia de otros enfoques de análisis de discurso dentro de las ciencias sociales, en que la estrategia general y principios en el proceso de configurar (abordar, plantear, construir y solucionar) el análisis es afín con el desarrollo alcanzado por las escuelas y corrientes de pensamiento sociológico contemporáneas para la comprensión y abordaje de lo social. De manera que el ASD como enfoque muestra perspectivas, métodos y procedimientos expresamente sociológicos de análisis de discurso. Ejemplos de estas perspectivas es el caso del análisis de discurso basado en la sociología del conocimiento, el 
análisis sociohermenéutico, el análisis reticular, el análisis posfundacional, el análisis socio-posestructural, el análisis del discurso (AD) como crítica social, el análisis etnográfico, los métodos cuantitativos en el $A D$, el análisis sociológico del sistema de discursos y el análisis de dispositivos establecido según la teoría social de Michel Foucault. Consúltese Herzog y Ruiz (2019).

La pertinencia de esta perspectiva reside en dos aspectos: uno teórico y otro metodológico. Desde el punto de vista teórico, para la Sociohermenéutica, los discursos son formas de práctica social que construyen distintos significados de lo social al representar espacios y conflictos sociales que permiten interpretar las relaciones sociales. Por tanto, los discursos - y su interpretación- remiten a "razones prácticas", en el sentido que le da a este concepto Bourdieu (1997), es decir, al conjunto de relaciones entre la posición y la toma de posición. En lo metodológico, el estudio de los discursos, tal como aquí se plantea, es una práctica de investigación relacional-reflexiva que posee una dimensión pragmática al utilizar textualidades empíricas para investigar regularidades sociales, centrándose en la tematización y en el encuentro de referencias contextuales a través de operaciones por analogías e interpretaciones locales.

\section{MÉTODOS Y MATERIALES}

El objeto de estudio se compuso de los discursos de 13 jóvenes bayameses residentes en un intervalo entre el Centro Histórico Urbano (calle 3 de Camilo Cienfuegos) y la periferia Norte (calle 24 de Ciro Redondo). Las fuentes de información (estos 13 jóvenes) se seleccionaron de acuerdo con un muestreo no probabilístico intencional, que se escogió según los criterios siguientes: ser jóvenes entre 19 y 30 años, que no tuvieran hijos, que vivan en núcleos familiares donde convivan al menos 3 generaciones en la misma vivienda. Los participantes en este estudio no fueron seleccionados bajo ningún criterio estadístico de representatividad, responden a que para la interpretación sociológica del discurso no es necesario un gran número de casos basta con examinar un número reducido de los discursos de los sujetos en posiciones sociales para inferir inductivamente.

Los jóvenes participantes fueron 6 mujeres y 7 hombres, con lo cual hubo una representación balanceada de los dos sexos, de igual manera entre los repartos de residencia, al estar presentes 7 en calle 3 del reparto Camilo Cienfuegos y 6 en calle 24 de Ciro Redondo. El grupo etario que predominó fue el 26 a 30 años con una representatividad del 54\%. El de menor representatividad fue el de 19 a 21 años.

Estos discursos se provocaron mediante la técnica de la entrevista. Para ello se diseñó una guía semiestructurada, compuesta por una serie de preguntas abiertas que permiten un margen de flexibilidad a partir de las respuestas de los sujetos. La estrategia metodológica se guió a través del marco analítico de Ruiz (2009), quien propone para el estudio sociológico del discurso la interrelación de tres niveles diferenciados de análisis: un nivel textual, un nivel contextual y un nivel interpretativo. Los dos primeros niveles responden a la caracterización y comprensión del discurso; y el tercero, corresponde a la explicación sociológica del mismo. A partir de esta perspectiva, se caracterizó el discurso mediante el procedimiento del análisis de contenido y se analizaron las estrategias discursivas adoptadas por los sujetos en este contexto. La interpretación sociológica se basó en el establecimiento del tejido relacional entre las posiciones discursivas a partir de las categorías relacionadas con la situación social emergente. 
El estudio confirmó la pertinencia del enfoque sociológico y la perspectiva sociohermenéutica para el análisis de los discursos sociales. Por otra parte, el análisis crítico del discurso permite adecuar la interpretación a la dimensión social del mismo, no sólo como práctica sino como una forma de construir ideología.

\section{RESULTADOS}

En relación con su ocupación solo un participante se estaba sin vínculo al estudio o al trabajo, condición estrechamente relacionada con la situación generada por las medidas para enfrentar la COVID- 19, se encuentra pendiente de retomar estudios. La mayor representatividad, respecto a la ocupación, se corresponde con los vinculados laboralmente (54\%), dentro de ellos los trabajadores estatales (38\%). Los trabajadores por cuenta propia representaron solo el $15 \%$ de los participantes en el estudio.

A partir del análisis de los discursos se rastrearon las principales configuraciones semánticas. Las redes semánticas están formadas por un conjunto de términos, de palabras, de vocablos, de ítems lexicales que se reiteran en un texto y que refieren a actores, relaciones, contextos, procesos, fenómenos, estados, objetos. Estos términos, los vocablos que se reiteran constituyen los nudos de esa red y configuran señales, marcas que orientan el sentido de la interpretación. Esos nudos de la red semántica se ubican en el núcleo de los modelos interpretativos de la realidad que emplean los hablantes. Consúltese Vasilachis de Gialdino (2007).

Pueden identificarse dos categorías fundamentales, que actúen como nudos en las redes semánticas presentes en los discursos juveniles, por su frecuencia de aparición en los jóvenes entrevistados: situación social y la COVID-19. La totalidad de los entrevistados reconocieron elementos que se pueden agrupar en ellas. Dentro de la situación social se incluyen las opiniones relacionadas con las prácticas, la socialización, la convivencia generacional, las relaciones personales y el aislamiento social. Para COVID-19 se tomaron los criterios relacionados con pandemia, percepción de riesgos, autocuidado y transmisión.

En relación con la percepción emerge un reconocimiento negativo como enfermedad; sin embargo, no todos están conscientes de la verdadera significación. Los principales cambios que identifican relacionados con las normas higiénicas son: cambios en las prácticas, las nuevas formas de socialización, el aislamiento y un mayor uso de las redes sociales. Sobre las iniciativas de los actores locales para enfrentar la pandemia son mayormente relacionadas por los entrevistados con el aislamiento social, el abastecimiento de productos, autocuidado, el cierre de espacios públicos y privados, la convivencia generacional, la distribución de labores en el hogar, la identificación de grupos vulnerables, los cambios en las prácticas, percepción del riesgo y el mayor uso de las redes sociales. Las opiniones referidas a la gestión de la información de los medios a los que acceden muestran resultados interesantes: todos acceden a la información desde diversas fuentes, con el predominio del uso de las redes sociales; la mayoría asocian las redes sociales al entretenimiento; reconocen la falsedad de la información que circula en las fuentes extranjeras y redes sociales; rechazo a los medios de información nacional justificado por la repetitividad y la saturación de la información.

Respecto a la COVID-19 se pudieron identificar percepciones de reconocimiento de la enfermedad; sin embargo, al analizar desde que posiciones (6 jóvenes) la relacionan con la preocupación por el aislamiento social y los cambios en las 
prácticas. Ejemplo de ello es esta opinión de una joven de 19 años plantea: "la situación que ha traído esta enfermedad me ha cambiado la vida. De todo lo que más me ataca, que me pone histérica es el aislamiento social. Nadie es capaz de imaginarse lo que es aguantar la gente en mi casa. Todo el mundo chocando, es verdad que es un infierno. Gracias que tenemos celulares". Otra joven expone: "yo realmente trabajo en la casa, así que con todo esto se terminaron mis saliditas a tomar y bailar [...] ir a la piscina, hacer ejercicios. Ahora solo me queda el teléfono y ver telenovelas. Yo sigo arreglando uñas, aunque la clientela ha bajado un poquito, pero siempre vienen. También esto ha traído algunos conflictos sobre todo con mi suegra que ahora está metida aquí en la casa el día entero opinando en todo. Ahora es que uno añora tener casa [...]" La forma de estructurar el discurso (en él cómo se dice) permite deducir una baja percepción de la enfermedad al sobrecargar el sentido a lo referido al aislamiento social, compartida por otras personas (clientas). Además, se distingue la conexión con otros discursos referidos a problemáticas sociales como la convivencia generacional, relaciones personales, la no disponibilidad de vivienda propia, etc.

Otro posicionamiento con respecto a la pandemia es lo relacionado con el cumplimiento de las normas higiénicas y los cambios ocasionados. Si bien la totalidad reconoce que sí hay cambios, los asocian con las prácticas, el aislamiento social, la socialización y el mayor uso de las redes. Categorías que evidencian una baja percepción acerca del riesgo de contraer la enfermedad, ejemplos: "de vez en cuando yo cumplo las medidas, por ejemplo, cuando hay que beber se bebe, pero en la casa"; "Mis rutinas son jugar fútbol, salir, bailar etc. Realmente no he cambiado mis rutinas, lo que ya no son al mismo ritmo, sigo haciendo algunas de ellas"; "Aunque nosotros seguimos haciendo ejercicio de forma clandestina, haciendo otras cosas que si nos cogen nos parten". Hay que destacar que de los 13 (11 jóvenes) reconocieron entre los principales cambios lo relacionado al autocuidado; pero resulta alarmante, cómo en las formas de estructurar los discursos, se evidencia el posicionamiento neutro en el cumplimiento de las medidas por parte de los entrevistados, al seguir realizando sus prácticas habituales.

Los distintos posicionamientos relacionados con las iniciativas locales y la protección de los grupos vulnerables son incompatibles con lo referido acerca del reconocimiento de la pandemia, el cumplimiento de las normas higiénicas y los cambios ocasionados. La primera incongruencia se encuentra en que reconocen los grupos vulnerables y aceptan las iniciativas locales para su protección; sin embargo, no asumen un rol dinámico participativo. Ejemplo de ello son estas expresiones: "en lo particular lo mío es limpiar y fregar, lo demás eso de hacer colas ni muerta y menos ahora con las matazones que hay"; "Yo realmente me la he pasado durmiendo, al punto que dicen que me van a salir escamas"; "pero hacer cola que va yo no tengo tiempo para eso"; "En mi casa son mi mamá y papá quienes se encargan de las labores, mi hermano y yo estudiamos y trabajamos, mis padres también pero esas son tareas que casi siempre hacen ellos"; "yo por lo general no tengo costumbre de hacer colas, en mi casa quien se dedica a esas cosas es mi abuelo y la sigue haciendo". En este posicionamiento se revela lo relacionado con la distribución de las labores en el hogar y la convivencia generacional, asunto que se vino a complejizar frente a los problemas de abastecimiento, el cierre de espacios públicos y privados, así como la demanda de un rol más participativo por parte de estos jóvenes. Es en este punto, donde sobresale una de las más importantes mediaciones que caracterizan a este grupo poblacional: el 
debilitamiento de los procesos de formación y expresión de un conjunto de valores que han signado a generaciones anteriores; y el empobrecimiento de la participación social en el interior de los hogares, esto último puede ser resultado de la dependencia de los adultos y la poca exigencia en la toma decisiones dentro de las familias.

Es importante destacar, desde la óptica sociológica, las redes semánticas que se establecen en estos discursos con respecto a los vocablos situación socialaislamiento-prácticas-redes sociales. Este posicionamiento discursivo es alto porque todos los entrevistados establecieron una línea de significación que atraviesa cada uno de estos componentes. Al relacionar la categoría situación social con prácticas de socialización habituales y aislamiento social se reveló en los discursos de (9 jóvenes) un posicionamiento negativo frente a la pandemia. En este sentido, no es el recrudecimiento de las necesidades materiales lo que los afecta, sino la interrupción de las prácticas lo que sobresale como un leitmotiv.

Como posiciones discursivas dominantes con respecto a la situación social emergente sobresalieron: el posicionamiento positivo al reconocer la importancia de las iniciativas locales, el acceso y la validez de la información que circula en los medios nacionales; el posicionamiento negativo en la identificación de los principales cambios relacionados con las normas higiénicas por los cambios en las prácticas, las nuevas formas de socialización y las consecuencias del aislamiento social. Además, con respecto a la gestión de la información a la que accede una posición discursiva dominante fue la importancia al uso de las redes sociales como entretenimiento, en rechazo a los medios de información nacional justificada por la repetitividad y la saturación de la información.

\section{CONCLUSIONES}

La sociedad cubana se enfrenta en la actualidad y en la perspectiva inmediata al impacto de la pandemia de la COVID-19 de elevada magnitud a nivel internacional. Este escenario coloca a la juventud cubana frente a un conjunto de retos que se derivan de su percepción y actitud para que se integre plenamente a ellos.

La aproximación socio-interpretativa a los discursos juveniles frente a la situación social emergente en el contexto de la COVID-19 arrojó una baja percepción de riesgo a contraer la pandemia.

Se identificaron como principales cambios asociados a las normas higiénicas la transformación en las prácticas cotidianas, las nuevas formas de socialización, el aislamiento y un mayor uso de las redes sociales. En la valoración acerca de la gestión de la información a la que acceden priorizan las fuentes extrajeras y resaltan la importancia al uso de las redes sociales como entretenimiento.

Se evidencia la percepción de que sí hay cambios (100\% de los de los jóvenes participantes) y estos se asocian a prácticas, aislamiento social, socialización y mayor uso de la red. Ello puede observarse en la frecuencia de aparición de palabras que conforman redes semánticas (en este caso referidos a situación socialaislamiento-prácticas-redes sociales).

La baja percepción del riesgo de contraer la enfermedad amerita un estudio más profundo en aras de aprehender elementos que incidan en ello (como pudieran ser imaginarios y representaciones asociados a juventud, salud u otros que motiven actitudes y prácticas irresponsables en el contexto de la COVID-19). 
Es posible apreciar, igualmente, una diferenciación respecto a valores traducidos en prácticas por generaciones de mayor edad, así como actitudes asociadas a la escasa participación en la distribución de responsabilidades en la vida doméstica, a lo interno de las familias.

Predomina el matiz positivo en el reconocimiento de las iniciativas locales, y al acceso y la validez de la información que circula en los medios nacionales. Entre los elementos que matizan las formas discursivas referidas a medios de información nacional, algunos entrevistados señalaron la repetitividad y la saturación de la información. Sería válido considerar una investigación que contemplara este tema, en aras de explorar formas de acercamiento a jóvenes de estos grupos etarios, desde los mencionados medios, con un lenguaje que pudieran percibir más cercano a sus formas de interacción.

\section{REFERENCIAS BIBLIOGRÁFICAS}

Alonso, L. E. (2013). La Sociohermenéutica como programa de investigación en sociología. Arbor, 189 (761): a035. doi: http://dx.doi.org/10.3989/ arbor.2013.761n3003.

Bourdieu, P. (1997). Razones prácticas sobre la teoría de la acción. Barcelona: Editorial Anagrama.

Conde, F. (2009) Análisis sociológico del sistema de discursos. Madrid: CIS, Cuadernos Metodológicos nำ43.

Domínguez, M. I. (2010). Oportunidades y restos para la integración social de la adolescencia y la juventud en Cuba hoy. En Domínguez, M. I. (Comp). Niñez, adolescencia y juventud en Cuba. Aportes para una comprensión social de su diversidad (pp. 99-112). La Habana: CIPS-UNICEF.

Domínguez, M. I. (2011). Políticas sociales hacia la juventud en Cuba: algunas evaluaciones y nuevos desafíos. Revista de Sociología, XVI (20), 28 -35.

Herzog, B.; Ruiz, J. (Eds.) (2019) Análisis sociológico del discurso. Enfoques, métodos y procedimientos. España: Universitat de València.

Morales, E. (Coord.) (2017). Identidad, cultura y juventud. La Habana: Instituto cubano de investigación cultural Juan Marinello.

Ramonet, I. (2020, 30 de mayo) Ante lo desconocido... la pandemia y el sistemamundo. Le Monde Diplomatique (edición chilena). Recuperado de https://www.lemondediplomatique.cl/ante-lo-desconocido-la-pandemia-y-elsistemamundo- por-ignacio-ramonet.html

Rego, I. (2014). Jóvenes cubanos en una sociedad que se transforma: algunos retos y oportunidades para la participación social. En CIPS: Cuadernos del CIPS. Experiencias de integración social en Cuba. La Habana: Publicaciones Acuario-Editorial Félix Varela, 114-133.

Ruiz, J. (2009, 26 de mayo) Análisis sociológico del discurso: métodos y lógicas En FQS Forum: Qualitative Social Research, 10 (2).

Ruiz, J.; Alonso, L.E (2019) Sociohermenéutica: fundamentos y procedimientos para interpretación sociológica de los discursos. En Herzog, B.; Ruiz, J. (Eds.) Análisis sociológico del discurso. Enfoques, métodos y procedimientos.

España: Universitat de València.

Vasilachis de Gialdino, I. (2007). Condiciones de trabajo y representaciones sociales. El discurso político, el discurso judicial y la prensa a la luz del 
análisis sociológico-lingüístico del discurso. Discurso \& Sociedad, 1(1), 148187. 